\title{
Multi-Mode and Single Goods Transportation Network Equilibrium Model Based on Super Network
}

\author{
Chen Ting \\ Beijing Information Technology College, Beijing, 100018 \\ hunter2011@foxmail.com
}

Keywords: Super Network; Multi-mode; Network Equilibrium.

\begin{abstract}
We expatiate and summarize the partition method about transportation mode, and then we analyze the function concept of generalized transportation cost in details in this paper. After the equilibrium model of regional transportation network and the algorithm of the multi-mode and single goods have been given, the problems of path search in the network are carefully studied. Based on path oriented model, we list two kinds of effective path search methods to specific problems in this paper, namely, the directional tree algorithm of efficient path and the directional hierarchical spatial reasoning algorithm of efficient path. Finally, we get the model and algorithm of the multi-mode transportation network equilibrium based on super network.
\end{abstract}

\section{Introduction}

With the development of the super network theory, people gradually realize that there are a large number of significant interactions between the basic networks which form the super network. For example, regional transportation network, information network and financial network are closely related with each other. The history of the goods information and cash flow situation stored in information network provide decision-making information for deciding the subsequent transport and cash flow. The transportation of goods inevitably occurs with the flow of funds and the generation of new information. And the flow of funds tends to affect the goods transportation and the generation of information.

Due to the complexity of network, the effect of the network policy has extensive influences, both in society and in economics. Just as the social justice problem and the fair use of the money problem brought about by the congestion charging policy.

To know the above features of the super network is useful for us to define the super network, and avoid possible bias in research.

We focus on the issue of network equilibrium while there are different transportation modes co-existed in regional transportation network, and make use of the super network and analyze the in-equation, finally get the corresponding network equilibrium model and algorithm in this paper. The core work of this paper is to convert multi-mode network model into the network model with employing single charge standard by setting reasonable generalized cost function. With transformation, the original complicated problems are simplified. The problems can be solved by using the commonly used algorithm of traffic flow distribution. Another focus of this paper is to add spatial price equilibrium principle among regional transportation network flows, and make network equilibrium reflect the intrinsic equilibrium driving force.

\section{Model and Algorithm of Traffic Flow Distribution under Multi-Mode Transportation}

The Setting of Generalized Sections' Cost Function Under the Multi-Mode Condition. Before analyzing generalized sections' cost function, while it exists multi-mode there. Firstly, the basic network parameters and constraints should be presented. Suppose that the network that under inspection is $G(N, L)$, in this network, $\mathrm{N}$ is a collection of nodes in network; $\mathrm{L}$ is the collection of sections in network. In the network, nodes are divided into three categories: the starting point of transport $r \in R$, the end of the transport $s \in S$, and ordinary nodes $i \in N-(R \cup S)$. The common 
sections in the network are represented by $a, b \in L$, and the general paths in the network are represented by $p, q \in P$. The above capital letters represent relevant elements collection. Suppose the flow on a section is $f_{a}$, the flow on the path $\mathrm{p}$ is $x_{p}$. Suppose That the number of sections and the number of paths in the network are respectively $n$ and $n p$, and the total flow on the sections and paths is represented by the following vectors $f=\left\{f_{a_{1}}, \cdots, f_{a_{n}}\right\}$ and $x=\left\{x_{p_{1}}, \cdots, x_{p_{n p}}\right\}$ respectively. Suppose that any O-D's flow on the ${ }^{r S}$ section ( $\mathrm{r}$ is the starting point, $\mathrm{s}$ is the end) is $d_{r s}$, and all paths on this section are represented by $P_{r s}$.

Among them, the equation (1) is the flow $d_{r s}$, which represents any O-D's flow on the

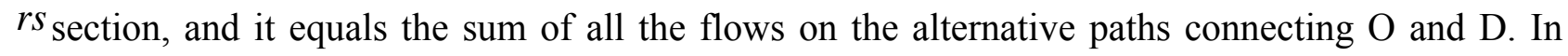
formula (2), $\delta_{a p}$ is the correlation coefficient between section a and path $\mathrm{p}$. If section a belongs to path p, we suppose $\delta_{a p}$ 's value is 1 ; Otherwise, 0 . Formula (2) means that the flow on any section is equal to the sum of flow that passed through this section. Formula (3) represents logic constraints of path flow, to ensure that the flow rate value is not negative. Based on the relationship between formula (2) and (3), it is easy to infer that the flow on any section of the path is nonnegative.

Next we will analyze the generalized transportation cost function related to specific section. Suppose that there are $\mathrm{H}$ kinds of cost indicators on any path to be used as a path choice of goods, and one of the possible path is h. Suppose $c_{h a}$ is the cost measurement of path $\mathrm{h}$, and is related with section $a$, then we can get the following equation,

$c_{h a}=c_{h a}(f) \quad \forall a \in L$

Here, we suppose the function $c_{h a}(f)$ is a continuous function of flow.

On the basis of formula (4), we can define the generalized transport cost for the road as $c_{a}=\sum_{h} c_{h a}(f) \quad \forall a \in L$

And generalized transport cost of section $c_{a}(f)$ is also a continuous function.

At the same time, the generalized transport cost for the path is $c_{p}=\sum_{a} \sum_{h} c_{h a} \delta_{a p} \quad \forall p \in P$

Based on the formula (4) and the relationship between the flow on the sections and the sum flow of paths on this section, the generalized transportation cost can be expressed as the function of section flow or paths flow of this section, as shown in the following equation,

$c_{p}=c_{p}(f)=c_{p}(x) \quad \forall p \in P$

There are many choices for the cost items of specific sections of the generalized transportation. We can choose according to the goods transported. Besides, the transportation time, transportation cost, the reliability of transportation and the opportunity cost of transportation, etc., can be the alternative indicators of cost. It is important to note that in formula (4), the assumed function has already considered the weights of different indicators. Therefore, there is no need to add the weight of each index in formula (5).

Before the model of multi-mode regional transportation network equilibrium is given, the path selection principle in the freight should be analyzed first. In practical application, both of the two principles are studied and put into practical use. For example, the famous software STAN freight distribution adapts the principle of the system optimal. System optimal principles play an important role in transportation policy analysis, while the user optimal principle has better explanation for decentralized decision in actual transportation market area. In STAN software, using the optimal system, for the author, is to achieve better convergence of model and the easier implementation of algorithm. Here, we will adapt a more realistic network transportation principle, namely, user optimal allocation principle.

User optimal allocation principle is equivalent to the utility maximization principle in economics, at the same time, it can be explained by Game Theory. This principle can be concretely embodied in 
the study of this paper. The user optimal principle refers to equilibrium state in the network, any actual chosen path between $\mathrm{O}$ and $\mathrm{D}$ has equal cost value, and this cost value is the smallest generalized transport cost value. For any path-p between the $\mathrm{O}$ and $\mathrm{D}$ on the $r s$ section, using user optimal allocation principle, we can get the result represented by the mathematical formula as $\begin{cases}c_{p}\left(x^{*}\right)-\pi_{r s}^{*}=0, & \text { if } x_{p}^{*}>0, \\ c_{p}\left(x^{*}\right)-\pi_{r s}^{*} \geq 0, & \text { if } x_{p}^{*}=0 .\end{cases}$

In formula (8), $\pi_{r s}$ represents the minimum generalized transport cost of all alternative paths between $\mathrm{O}$ and $\mathrm{D}$ on the section $r s$. The superscript "*" represents the quantity of corresponding values, namely, the network equilibrium values.

Suppose O - D can exert elasticity on the freight flow on the section $r s$, here we can get the following function,

$\pi_{r s}=\pi_{r s}(d) \quad \forall r s$

Suppose the function that formula (9) represents a reduction function related to O - D and the freight flow between $r s$, so the smaller the value of $\pi_{r s}$, the greater the value of $d_{r s}$. In this equation, $d$ is a dimensional vector made up of $d_{r s}, \forall r s$. $|r s|$ represents the total number on $\mathrm{O}$ $\mathrm{D}$ network. The elastic demand assumptions make the freight flow on rs section by O-D, namely, $d_{r s}$ be changed into a variable. The optimal distribution principle under elastic demand can be represented as the following formula

$\begin{cases}c_{p}\left(x^{*}\right)-\pi_{r s}\left(d^{*}\right)=0, & \text { if } x_{p}^{*}>0, \\ c_{p}\left(x^{*}\right)-\pi_{r s}\left(d^{*}\right) \geq 0, & \text { if } x_{p}^{*}=0 .\end{cases}$

The following are inequality model got correspondingly according to the formula (10) and formula (8).

Based on formula (10), we can get,

$\left(c_{p}\left(x^{*}\right)-\pi_{r s}\left(d^{*}\right)\right) \times x_{p}^{*}=0$

$c_{p}\left(x^{*}\right)-\pi_{r s}\left(d^{*}\right) \geq 0$

From the non-negative constraints of path flow (3) and the formula (12), we can get, $\left(c_{p}\left(x^{*}\right)-\pi_{r s}\left(d^{*}\right)\right) \times x_{p} \geq 0$

Further combining with formula (11), the following can be got, $\left(c_{p}\left(x^{*}\right)-\pi_{r s}\left(d^{*}\right)\right) \times\left(x_{p}-x_{p}^{*}\right) \geq 0 \quad \forall p \in P_{r s}$

After we sum up all the paths' value using the formula (13), we can get, $\sum_{r s} \sum_{p \in P_{r s}}\left(c_{p}\left(x^{*}\right)-\pi_{r s}\left(d^{*}\right)\right) \times\left(x_{p}-x_{p}^{*}\right) \geq 0$

We put formula (1) into the above formula, and finally we can get,

$\sum_{r s} \sum_{p \in P_{r s}} c_{p}\left(x^{*}\right) \times\left(x_{p}-x_{p}^{*}\right)-\sum_{r s} \pi_{r s}\left(d^{*}\right) \times\left(d_{r s}-d_{r s}^{*}\right) \geq 0$

Thus the corresponding variational inequality of formula (10) can be got, this variational inequality constraint setting can be seen as follows,

$\mathrm{K}$ is the point represented by $(\mathrm{x}, \mathrm{d})$, and the corresponding path flow and the flow between $\mathrm{O}$ and $\mathrm{D}$ meet with the formula (1), (2), (3).

The above derivation process is reversible, therefore the variational inequality model is equal to formula (10).

For formula (8), there is a similar derivation, and the final VI model is

$\sum_{r s} \sum_{p \in P_{r s}} c_{p}\left(x^{*}\right) \times\left(x_{p}-x_{p}^{*}\right) \geq 0$

The corresponding constraint set $\mathrm{K}^{\prime}$ equals $\mathrm{x}$, and the corresponding path flow meet with the formula (5.1), (5.2), (5.3). 
If we use formula (2) can convert the flow distribution model represented by formula (15) and (16) into VI which can be got based on the path flow. The corresponding transformation results can be shown as the following equation respectively,

$$
\begin{aligned}
& \sum_{a} c_{a}\left(f^{*}\right) \times\left(f_{a}-f_{a}^{*}\right)-\sum_{r s} \pi_{r s}\left(d^{*}\right) \times\left(d_{r s}-d_{r s}^{*}\right) \geq 0 \\
& \sum_{a} c_{a}\left(f^{*}\right) \times\left(f_{a}-f_{a}^{*}\right) \geq 0
\end{aligned}
$$

The corresponding constraint setting should also be revised correspondingly.

For the VI model (15) or (17) under the condition of elastic demand, although the VI influenced function is a continuous function, because there is no limitation of the constraint set, the existence of the solution can't be got directly. A related theorem is given below to make sure that there is a solution to VI model (15) or (17).

\section{Multi-mode Transportation Network Equilibrium Model and Algorithm Based on the Super network}

First of all, we analyze the decision-making behavior of the distributors in the transportation network supply node.

Because we consider that the regional transportation problem of single goods in this paper. We assume that there is a distributor in each starting node. Under the decision-making principle of benefit maximization, the distributor will determine the total supply of its goods (namely, the purchase quantity of the goods), the transportation route of goods, and specific transportation capacity of each line. We set the goods purchase quantity of distributor in Starting point $r$ as $s u_{r}$, and the goods purchase cost function of distributor as $g_{r}(s u)$. We use $|R|$ to indicate the number of the elements in a collection. su is the $|R|$ dimensional vector which is made up of all goods purchase quantity $s u_{r}, r \in R$. The form of goods purchase cost function $g_{r}(s u)$ can explain that goods purchase cost of distributor in $r$ is not only related to the local supply of goods, but also related to goods supply quantity of other places. This reflects the competitive relationship of the goods supply with the same kind around the place. Further we set the goods purchase cost function with separation characteristics, that is to say, it can be expressed as

$$
g_{r}(s u)=g_{r 1}\left(s u_{r}\right)+g_{r 2}\left(s \hat{u}_{r}\right)
$$

In the formula (19), after we remove elements $s u_{r}, s \hat{u}_{r}$ is the vector which is composed by other elements in the vector $s u$. The goods purchase cost function with the form of separation will provide the convenience for establishing VI model in later illustration. We set $d_{r s}$ as the total quantity of goods which the distributor in $r$ provides the consumer in $s$. We can get the following formula from the conservation relations of flow.

$\sum_{s} d_{r s}=s u_{r} \quad \forall r \in R$

In addition to the pay of goods purchase cost, we assume that the distributor also needs to advance the goods transportation cost. The transportation cost here refers to the generalized transportation cost of the route. Under the condition of determining the network flow model, if the distributor chooses the route $p \in P_{r s}$, we set corresponding transportation cost of goods unit as $c_{p}(x), p \in P_{r s}$. We can get that the total payment with transportation cost of distributor in $\mathrm{r}$ is $C_{r}(x)=\sum_{p \in P_{r}} C_{p}(x) x_{p}$. From the definition of $C_{r}(x)$, we can get

$$
\frac{\partial C_{r}(x)}{\partial x_{p}}=c_{p}(x)+\sum_{q \in P_{r}} \frac{C_{q}(x)}{x_{p}} x_{q} \quad \forall p \in P_{r}, r
$$

In the state of equilibrium network, the distributor will always choose the minimal route of marginal unit freight as far as itself is concerned. When the network does not reach the equilibrium 
state, the distributor will always choose the minimal route of marginal unit freight as far as itself is concerned to transport. The following model will reflect the characteristic.

At the same time, we assume that the distributor in $r$ would set the price $p r_{r s}$ for the goods which transport to $\mathrm{S}$ position. Then sales revenue with anticipation of the distributor in $\mathrm{r}$ is $\sum_{s} p r_{r s} d_{r s}=\sum_{s} \sum_{p \in P_{r s}} x_{p} p r_{r s}$.

We make a comprehensive analysis of the decision-making index of the distributor in $r$ mentioned above, and we can get the the optimal objective function, which is max

$\sum_{s} \sum_{p \in P_{r s}} x_{p} p r_{r s}-\sum_{p \in P_{r}} c_{p}(x) x_{p}-g_{r 1}\left(s u_{r}\right)-g_{r 2}\left(s \hat{u}_{r}\right)$

The corresponding constraint equations are (1), (3) and (21), we will give them again as follows.

$\sum_{s} d_{r s}=s u_{r} \quad \forall r \in R$

$\sum_{p \in P_{r s}} x_{p}=d_{r s} \quad \forall r \in R, s \in S$

$x_{p} \geq 0 \quad \forall p \in P$

From the flow relations of (22b) and (22c), we know that the following formula gets to work.

$\frac{\partial g_{r}(x)}{\partial x_{p}}=\frac{\partial g_{r}(s u)}{\partial s u_{r}}=\frac{\mathrm{d} g_{r 1}\left(s u_{r}\right)}{\mathrm{d} s u_{r}}=\frac{\partial g_{r 1}(d)}{\partial d_{r s}}=\frac{\partial g_{r 1}(x)}{\partial x_{p}}$

By using the formula (35), we can get the VI model of the corresponding problem (22) as follows.

$\left.\sum_{r} \sum_{s} \sum_{p P_{s}} \frac{\partial g_{r}\left(x^{*}\right)}{\partial x_{p}}+c_{p}\left(x^{*}\right)+\sum_{q \in P_{p}} \frac{\partial c_{q}\left(x^{*}\right)}{\partial x_{p}} x_{q} x^{*}-p r_{r s}\right) \times\left(x_{p}-x_{p}^{*}\right) \geq 0 \quad \forall x \in R_{+}^{n p}$

The formula (24) has a good explanation of economics. That is to say, when the distributor provides freight, the sum of the margin purchase cost of unitized goods as far as the distributor itself is concerned and unitized margin transportation cost is greater than or equal to its corresponding price for the goods. While the sum of the margin purchase cost of unitized goods as far as the distributor itself is concerned and unitized margin transportation cost is less than its corresponding price for the goods, it shows that there is no supply.

Next, we will analyze the consumer's decision-making behavior of demand node in regional transportation network. We set the goods price of demand node $\mathrm{s}$ position as $p r_{s}$, then the corresponding demand quantity is

$d_{s}=d_{s}\left(p r_{s}\right) \quad \forall p r_{s} \in R_{+}$

Then we will give network equilibrium conditions of demand node s position. As far as the consumer in demand node s position is concerned, the following formula can get to work for all O-D pair.

$p r_{r s} \begin{cases}=p r_{s}^{*}, & \text { if } d_{r s}^{*}>0 \\ \geq p r_{s}^{*}, & \text { if } d_{r s}^{*}=0\end{cases}$

At the same time, the following formula can also get to work for all demand nodes s.

$d_{s}\left(p r_{s}^{*}\right) \begin{cases}=\sum_{r} d_{r s}^{*}, & \text { if } p r_{s}^{*}>0 \\ \leq \sum_{r} d_{r s}^{*}, & \text { if } p r_{s}^{*}=0\end{cases}$

The meaning of formula (26) is that when the price which is formulated by the distributor in the $r$ position for the goods transported to the demand node $s$ is not greater than the price which the consumer is willing to pay in the demand node $\mathrm{s}$, the consumer in s position will consume the goods from $r$. The meaning of formula (27) is that when the price which the consumer is willing to pay in s position is positive, the actual consumption quantity in s position is equal to the actual demand quantity of the goods in s position. 
We set that $p \tilde{r}$ is $|S|$ dimensional vector composed of all $p r_{s}$ and $\forall s$. We use corresponding variational inequalities to express formula (26) and formula (27). And the result can be seen as follows.

$$
\begin{aligned}
& \sum_{r} \sum_{s}\left(p r_{r s}-p r_{s}^{*}\right) \times\left(d_{r s}-d_{r s}^{*}\right)+\sum_{s}\left(\sum_{r} d_{r s}^{*}-d_{s}\left(p r_{s}^{*}\right)\right) \times\left(p r_{s}-p r_{s}^{*}\right) \geq 0 \\
& \forall(d, p \widetilde{r}) \in R_{+}^{|r s|+|s|}
\end{aligned}
$$

We make VI model (25) and model VI (28) be added together, so we get

$$
\begin{aligned}
& \sum_{r} \sum_{s} \sum_{p \in P_{r s}}\left(\frac{\partial g_{r}\left(x^{*}\right)}{\partial x_{p}}+c_{p}\left(x^{*}\right)+\sum_{q \in P_{r}} \frac{\partial c_{q}\left(x^{*}\right)}{\partial x_{p}} x_{q}^{*}-p r_{r s}\right) \times\left(x_{p}-x_{p}^{*}\right) \\
& +\sum_{r} \sum_{s}\left(p r_{r s}-p r_{s}^{*}\right) \times\left(d_{r s}-d_{r s}^{*}\right)+\sum_{s}\left(\sum_{r} d_{r s}^{*}-d_{s}\left(p r_{s}^{*}\right)\right) \times\left(p r_{s}-p r_{s}^{*}\right) \geq 0
\end{aligned}
$$

$$
\forall(x, d, p \widetilde{r}) \in R_{+}^{n p+|r s|+|s|}
$$

By using the flow equation $(22 \mathrm{c})$ to simplify the above formula further, we can get

$$
\begin{aligned}
& \sum_{r} \sum_{s} \sum_{p \in P_{r s}}\left(\frac{\partial g_{r}\left(x^{*}\right)}{\partial x_{p}}+c_{p}\left(x^{*}\right)+\sum_{q \in P_{r}} \frac{\partial c_{q}\left(x^{*}\right)}{\partial x_{p}} x_{q}^{*}-p r_{s}^{*}\right) \times\left(x_{p}-x_{p}^{*}\right) \\
& +\sum_{s}\left(\sum_{r} \sum_{p \in P_{r s}} x_{p}^{*}-d_{s}\left(p r_{s}^{*}\right)\right) \times\left(p r_{s}-p r_{s}^{*}\right) \geq 0
\end{aligned}
$$

$$
\forall(x, p \widetilde{r}) \in R_{+}^{n p+|S|}
$$

The formula (30) is the final network equilibrium VI model which is got after we integrate the decision-making of the distributor and the consumer.

We will give the solving steps of modified projection method about VI model (30) in the following content.

\section{Conclusion}

This paper makes the extension of regional transportation network model about single mode and single goods from diverse angles about means of transport. First, we expatiate and summarize the different classification methods about means of transport, and then we generally analyze the function concept of generalized transportation cost. The establishment of the concept with generalized transportation cost makes it possible for establishing regional transportation network equilibrium model based on multi-mode and single goods, and makes the transportation network model with simple single mode and single goods be able to be smoothly extended to the scene with multi-mode and single goods. At the same time, we analyze path search problems in network construction problem and network about the multi-mode transport. In the paper, we give two kinds of effective path search methods based on the model solution of path, that is to say, the directional tree algorithm of efficient path and the directional hierarchical spatial reasoning algorithm of efficient path. Finally by analyzing spatial price equilibrium principle with the introduction of the decision-makers' behaviors in regional transportation network we give multi-mode transportation network equilibrium model and algorithm based on super network.

\section{Reference}

[1] Nagurney, A. Network Economics: A Variational Inequality Approach. Second and revised edition, Kluwer Academic Publishers, Dordrecht, The Netherlands, 1999.

[2] Nagurney, A. Navigating the Network Economy. OR/MS Today, 2000, June, 7.

[3] Takayama, T. and G. G. Judge. Spatial and temporal price and allocation models. North-Holland, Amsterdam, The Netherlands, 1971. 
[4] Zhao, L. Variational Inequalities in General Equilibrium: Analysis and Computation. Division of Applied Mathematics, Brown University, Providence, Rhode Island, 1989.

[5] Zhao, L., and S. Dafermos. General Economic Equilibrium and Variational Inequalities. Operations Research Letters 1991, 10, 369-376.

[6] Zhao, L. and A. Nagurney. A Network Formalism for Pure Exchange Economic Equilibria. In Network Optimization Problems: Algorithms, Complexity and Applications, pp. 363-386, D. Z. Du and P.M. Pardalos, editors, World Scientific Press, Singapore, 1993. 